\title{
$\checkmark$ Research Square \\ Quantized wrinkles and fracture of stiff membranes on soft films
}

\section{Yancheng Meng}

Nanjing University of Aeronautics and Astronautics

\section{Henggao Xiang}

Nanjing University of Aeronautics and Astronautics

Jianqiang Zhang

Nanjing University of Aeronautics and Astronautics

Zhili Hu

Nanjing University of Aeronautics and Astronautics

Jun Yin

Nanjing University of Aeronautics and Astronautics

Liqiang Li

Tianjin University https://orcid.org/0000-0001-8399-3957

\section{Zhuhua Zhang}

Nanjing University of Aeronautics and Astronautics https://orcid.org/0000-0001-6406-0959

Wanlin Guo ( $\nabla$ wlguo@nuaa.edu.cn )

Nanjing University of Aeronautics and Astronautics https://orcid.org/0000-0002-2302-8044

\section{Article}

Keywords:

Posted Date: January 14th, 2022

DOI: https://doi.org/10.21203/rs.3.rs-1212340/v1

License: (c) (i) This work is licensed under a Creative Commons Attribution 4.0 International License.

Read Full License 


\section{Abstract}

Stiff membranes on soft substrates may wrinkle and fold during compression ${ }^{1-11}$, but the strong postbuckling nonlinearity 3,12 and the propensity of overall bending of these systems $s^{4,9,11}$ under large compression make the intriguing morphological evolution ill-controlled and less understood. Here, we present a simple peeling strategy that controllably makes stiff nanomembranes on soft microfilms wrinkled, then folded with a preset period, and ultimately fractured into regular ribbons. The fold and fracture periods exhibit a quantized, stepped dependence on the microfilm thickness, with the period doubled per step. The controlled wrinkle-to-fold-to-fractures transitions can be quantified by both computations and a scaling law, showing generality to different forms of compressive loading. This quantized wrinkle evolution deepens our understanding of complex behaviors of such natural and artificial systems as cerebral cortexes, skins, and coating materials, and opens a way to advanced manufacturing by fracturing large-area nanomembranes into uniformly shaped microflakes.

\section{Main Text}

Our artificial mechanical systems, chips and computers need enough stiffness and strength, while the bio-systems and natural intelligent brain are soft with wrinkles and folds. When stiff meets soft, stiff/soft bilayers always lose their stability ${ }^{1}$ and form a variety of morphological instability modes ${ }^{2-4}$, such as wrinkles $^{1,5-8}$, localized folds ${ }^{2,9-11}$, and ridges ${ }^{12,13}$, during compression. Such an evolving bilayer system plays a key role in working rationale of bio-systems ranging from ageing skins, lung surfactants to complex and incomprehensible structures of cerebral cortexes ${ }^{14-17}$, and heralds a wealth of opportunities for applications in advanced materials and devices ${ }^{18-20}$.

Regular wrinkles are the primary instability mode, especially at high stiff/soft modulus ratio and large thickness contrast ${ }^{3}$. Secondary instability occurs in the period-wrinkling membranes upon further compression ${ }^{2}$, manifested mainly as winkle-to-fold transitions driven by stress localization. This transition results in double and quadruple periods in the stiff membrane on a much thicker soft film for energy minimization ${ }^{9}$. The dependence of instability modes on the modulus ratio and thickness ratio of stiff/soft bilayers have been systematically analyzed in the past decade ${ }^{3,21,22}$. Under higher compression, buckling folds collapse, resulting in highly concentrated stress at the collapsed bottoms. However, the strong nonlinearity of post-buckling deformation makes it challenging to controllably induce and manipulate the fold instability in large scale, not to mention potential applications. Here, we show that regular wrinkles formed in stiff nanomembranes bound onto soft microfilms can be well-controlled to fold period-singly, doubly or quadruply by peeling the microfilms perpendicularly from a rigid substrate. With these wrinkle-to-fold transitions of multiple routes, a range of large-area nanomembranes can be fractured into arrays of micro-ribbons and even micro-flakes with characteristic sizes set by the fold period. More interestingly, the fold period and the ribbon width exhibit a quantized dependence on the local strain in the soft microfilm, which turns out to be a general behavior to different forms of loading. Our results reveal a previously unrecognized attribute of ubiquitous wrinkles in soft systems. 
The stiff/soft bilayer was fabricated by placing a polydimethylsiloxane (PDMS) microfilm on a rigid substrate and then exposing to oxygen plasma to create a stiff $\mathrm{SiO}_{1.8}$ coating layer (Fig. 1a), a process similar to a previous report ${ }^{23}$. The thickness of the soft PDMS film, $t_{\mathrm{s}}$, ranges from 20 to $300 \mu \mathrm{m}$, while that of the $\mathrm{SiO}_{1.8}$ membrane, $t_{\mathrm{m}}$, is ranged in $30-281 \mathrm{~nm}$. The Young's modulus $E_{\mathrm{m}}$ of the $\mathrm{SiO}_{1.8}$ layer is 30 $\mathrm{GPa}$, about four orders of magnitude higher than that $\left(E_{\mathrm{s}}=1.8 \mathrm{MPa}\right)$ of the PDMS microfilm. Stretching such a stiff/soft bilayer in one direction fractures the $\mathrm{SiO}_{1.8}$ layer perpendicularly into strips, which simultaneously form wrinkles due to compression induced by the Poisson effect (Fig. S3). The intrinsic periodic length $\lambda_{0}$ at the threshold of the wrinkling instability is proportional to the thickness of the stiff membrane $t_{\mathrm{m}}$, expressed as $\lambda_{0}=A t_{\mathrm{m}}$, where $A$ is a constant related to elastic properties of the bilayer (see $\mathrm{SI})$. Enhancing the stretch makes the strips more compressed, driving wrinkle-to-fold transitions and foldcollapses. Yet, the double and quadruple folds as well as the corresponding fold-collapses appear randomly under the uniaxial stretch (Fig. S3), and no control on the instability modes has been achieved thus far.

We achieve the morphological control by perpendicularly peeling the bilayer from the rigid substrate (Fig. 1a). The bilayer under perpendicular peeling is compressed in its inner side, along with a gradient from the flat, unpeeled region to the bent region. Accordingly, wrinkles, double folds, quadruple folds, and quadruple fold-collapses simultaneously appear in respective regions of the stiff membrane, as shown in Fig. $1 \mathrm{~b} \otimes$ for a bilayer with $t_{\mathrm{s}}=112 \mu \mathrm{m}$ and $t_{\mathrm{m}}=155 \mathrm{~nm}$. The instability modes encoded by local curvatures of the bilayer form a stark contrast to a randomly occurred, single instability mode under the uniaxial stretch (Fig. S3). More interestingly, the quadruple fold-collapse holds a deep-set, steep bottom (i, Fig. 1c and Fig. S4) that has been partially cracked at the apex (ii, Fig. 1c) due to highly concentrated stress thereof ${ }^{24}$. Membrane fracture occurs at the collapsed bottom as the local curvature further increases (iii, Fig. 1c). As such, the fracture route is predefined along the fold line, and, thus, strictly straight and controllable, in contrast to normal fracture that is dominated by structural defects. As the peeling proceeds, the wrinkles dynamically evolve and the quadruple fold-collapses occur progressively throughout the whole bilayer until the bilayer is completely peeled off, leaving the membrane evenly fractured with a pattern period of $4 \lambda_{0}$ (Fig. $\left.1 \mathrm{~d} \mathbb{\mathrm { d }}\right)$.

The fracture pattern is deterministically controlled by the thickness of the soft microfilm $t_{\mathrm{s}}$. For two bilayers with the same $t_{\mathrm{m}}=155 \mathrm{~nm}$ as above, we find that wrinkles in the one with $t_{\mathrm{s}}=33 \mu \mathrm{m}$ evolves only into single-period fold-collapses, resulting in a fracture pattern with a period of $1 \lambda_{0}$ (Fig. $1 \mathrm{~b} \mathbb{\mathrm { b }}$ ). Note that the single-period folds and collapses are a missing mode in previous theory and experiments ${ }^{2,9}$. Our peeling method can access this mode because the wrinkles experience a localized evolution driven by an extremely high compressive strain that is mediated by $t_{\mathrm{s}}$. The other bilayer with $t_{\mathrm{s}}=90 \mu \mathrm{m}$ is fractured with a $2 \lambda_{0}$ period due to its double-period folds and ensuing fold-collapses (Fig. 1b $\mathbb{b l}$ ). Thus, three distinct buckling evolution routes starting from wrinkles and ending with fracture emerge: wrinkles to single-folds and to single-fold-collapses as route 1 (Fig. 1b区); wrinkles to double-folds, and to double-fold-collapses as route 2 (Fig. 1bखl); wrinkles to double-folds, quadruple-folds, and to quadruple-fold-collapses as route 3 
(Fig. $1 \mathrm{~d} \rrbracket$ ). At a given $t_{\mathrm{m}}$, which evolution route will be taken by a peeling bilayer is controlled by $t_{\mathrm{s}}$, so does the number of buckling modes (Fig. S5).

Extensive measurements on a series of stiff/soft bilayers reveal that all the acquired fracture patterns fall into three periods following the three routes, i.e., $1 \lambda_{0}, 2 \lambda_{0}$, and $4 \lambda_{0}$ (Figs. 1 e and S6). Specifically, the bilayers with $t_{\mathrm{m}}=155 \mathrm{~nm}$ exhibits a $1 \lambda_{0}$ fracture period when $t_{\mathrm{s}} / \lambda_{0}<2.5$, a $2 \lambda_{0}$ period when $2.5<t_{\mathrm{s}} / \lambda_{0}$ $<9.5$, and a $4 \lambda_{0}$ period when $t_{\mathrm{s}} / \lambda_{0}>9.5$ (Fig. 1e). Further increasing $t_{\mathrm{s}} / \lambda_{0}$ over 40 can result in an $8 \lambda_{0}$ fracture period (Fig. S7), although the corresponding bilayers are too thick to realize a steady peeling process. This stepped thickness dependence of fracture periods can be viewed as a discretization of wave-like wrinkles upon large compression, much like quantized energy of electronic wavefunctions induced by spatial confinement. The critical $t_{\mathrm{s}} / \lambda_{0}$ for the transitions between distinct periods, denoted as $t_{\mathrm{s} N} / \lambda_{0}$ with $N=1,2$, and 3 in Fig. $1 \mathrm{e}$, are constants. As $\lambda_{0}$ is proportional to $t_{\mathrm{m}}, t_{\mathrm{s} N}$ linearly increases with $t_{\mathrm{m}}$ as well, consistent with our extensive measurements on bilayers with different $t_{\mathrm{m}}$ (Fig. S6). Accordingly, the ribbon width $W$ and $t_{\mathrm{m}}$ are linearly related by $W=n A t_{\mathrm{m}}(n=1,2,4)$, which again agrees with our experiments (Fig. S8). Of technical importance is that $W$ is as small as $8 \mu \mathrm{m}\left(2 \lambda_{0}\right)$ when peeling a bilayer with $t_{\mathrm{m}}=42 \mathrm{~nm}$ and $t_{\mathrm{s}}=76 \mu \mathrm{m}$ (Fig. S8). Note that the peeling angle of no less than $90^{\circ}$ is crucial to the quantized fractures in the membranes. Otherwise, the bending-induced compression does not suffice to trigger the wrinkle-to-fold transitions; instead, nonperiodic fracture appears in the peeled part of the bilayer due to the in-plane stretch applied for peeling (Fig. S9).

The stepped thickness dependence of fracture patterns can be understood by a continuum model, in which the soft microfilm under peeling is approximated as a cantilever beam with large deflection ${ }^{25}$ (see $\mathrm{SI})$. According to this model, the strain increases with moving away from the free end and reaches a maximum at the clamped point. Yet, the beam model cannot correctly describe the strain distribution in the region near the clamped point since the strain must be continuous across this point. As such, the practical maximum strain point deviates from the clamped end by a certain distance. This deviation indeed exists in our experimental samples under peeling, with magnitudes in a range $0.12 t_{\mathrm{s}}-0.18 t_{\mathrm{s}}$ (see SI) and an average of $0.16 t_{\mathrm{s}}$. Therefore, we correct our model by taking this deviation into account for determining the maximal strain (see $\mathrm{SI}$ ).

The bending-induced strain in the soft microfilm is transferred into the stiff membrane. Here, we focus on the membrane region where the fracture occurs due to the maximal strain. For a fold-collapsed membrane with a period of $n \lambda_{0}(n=1,2,4)$, the total strain within a period is localized to the collapsed region and can be assessed through integrating the strain over the same period length of the microfilm prior to the buckling (Fig. 2a). This integrated strain $\Delta_{n}$ is just a shortened length of the microfilm normalized by $\lambda_{0}$. Since the overall compressive strain in the microfilm is proportional to $t_{\mathrm{s}}^{-1 / 2}$ (see SI), $\Delta_{n}$

scales as

$$
\Delta_{n}=2.28 n \sqrt{\frac{f_{0}}{\overline{E s}_{s}}},
$$


where $=E_{\mathrm{s}} /\left(1-\mu_{\mathrm{s}}^{2}\right), f_{0}$ is the adhesive force per unit width between the bilayer and the substrate, and $E_{\mathrm{s}}$ and $\mu_{\mathrm{s}}$ are the Young's modulus and Poisson ratio of the soft microfilm, respectively. The details on deriving the Eq. 2 are provided in SI. Based on experimentally measured critical $t_{\mathrm{s}}$ (Fig. 1e), the fitted critical $\Delta_{n}$ reads $=\left(c_{n} t_{\mathrm{m}}{ }^{1 / 2}\right) / \lambda_{0}$ for the $n \lambda_{0}$-period fracture, where $c_{n}=10^{-3 / 2} \mathrm{~m}^{1 / 2}$ (see SI). Surprisingly, is a constant independent of $n$, thus denoted as $\Delta^{\mathrm{C}}$, for all the bilayers with a given $t_{\mathrm{m}} \cdot \Delta^{\mathrm{C}} \cong 0.9$ when $t_{\mathrm{m}}=$ $155 \mathrm{~nm}$ (Fig. S10), close to 0.85 from experimental measurements on three bilayers with $t_{\mathrm{m}}=155 \mathrm{~nm}$ and $t_{\mathrm{S}}=t_{\mathrm{S} N}(N=1,2$ and 3 , see Fig. $2 \mathrm{~b}$, olive spheres). These results enable us to constitute a criterion, $\Delta_{n} \geq 0.9$, for the fracture of $\mathrm{SiO}_{1.8} / \mathrm{PDMS}$ bilayers with a period of $n \lambda_{0}$. For example, the bilayers with $2.5<t_{\mathrm{s}} / \lambda_{0}<9.5$ can only be fractured with a period of $2 \lambda_{0}$, because its $\Delta_{1}$ is below 0.9 while $\Delta_{2}$ meets this criterion (Fig. 2b).

According to the expressions of $\Delta_{n}$ and $\Delta^{c}, t_{s N}$ can be expressed as $t_{s N}=2^{2 N} C t_{m}$, where $C$ is a constant of . This expression dictates $t_{\mathrm{s} N} / t_{\mathrm{s}(N-1)} \equiv 4$ for the thickness-dependent fracture of stiff/soft bilayers, that is a doubling of the fracture period requires a fourfold increase of $t_{\mathrm{s}} / \lambda_{0}$. This scaling law is in agreement with the extensive experimental results (Figs. 1e and Fig. S6) and represents a new mechanical attribute of stiff/soft bilayers that can be wrinkled and folded under compression.

The buckling evolution in the bilayers induced by mechanical peeling is further supported by finite element simulations (see details in SI). Figure 2c presents the simulation results of a stiff/soft bilayer with $t_{\mathrm{m}}=155 \mathrm{~nm}$ and $t_{\mathrm{s}}=60 \mu \mathrm{m}$. When the bilayer is slightly bent, wrinkles appear in its compressed side. The wrinkles gradually evolve into double folds as the bilayer is more bent, similar to the experimental results and amenable to the above criterion. The calculated von Mises stress in the stiff membrane exhibits a relatively regular distribution for the wrinkles (Fig. 2d), but becomes scattered with a pronounced peak when the double folds appear (Fig. $2 \mathrm{~d}$ and inset). The peak lies at the fold bottom that is deviated from the very peeling front, just as what is observed in our experiments. This deviation further justifies the correction of our above continuum model. Thus, the ultrahigh stress at the fold bottom leads to eventual fracture of stiff membranes. The simulated results are robust against changing $t_{\mathrm{s}}$ in a wide range 20-300 $\mu \mathrm{m}$ and agree with experiments in terms of the buckling period (Figs. 1e and Fig. S11) and its evolution (Fig. S12).

The quantized folds and fracture in the stiff/soft bilayers are not unique to the peeling method but a general behavior to any form of loading that can effectively compress the bilayers. Regarding the uniaxial stretch method that compresses the bilayers by Poisson effect (Fig. 3, inset), the local fold-collapse and facture period in the most compressed region still follows a stepped dependence on the local compressive strain $\varepsilon$, although buckling modes occur randomly in the whole bilayer (Fig. S3). If we define an equivalent strain as (see $\mathrm{SI}$ ), the stepped dependence becomes uniform for all the bilayer with different $t_{\mathrm{m}}$ and $\lambda_{0}$ (Fig. 3). Moreover, the critical for the transitions between distinct fracture periods nearly reproduce those determined from above peeling experiments, as compared by the black and gray lines in Fig. 3. Notably, the fold-collapse periods collected from refs. 8 and 22, where the bilayers are 
directly squeezed, can also be quantified by our scaling law. These results further verify the intrinsic behavior of quantized wrinkles in stiff/soft bilayers.

In 2008, Pocivavsek et al. reported that wrinkles can evolve into a local fold in a soft polyester film resting on water under lateral compression, but into double or quadruple folds when the film is on a gel substrate $^{2}$. Later, a model analysis based on nonlinear oscillator dynamics unraveled that the wrinkle-tofold transitions were triggered by a period-doubling bifurcation ${ }^{9}$. Subsequent finite element simulations and analyses underscored the role of a relatively small modulus ratio of stiff/soft bilayers in inducing the buckling folds rather than outward ridges ${ }^{3,21,26}$. Similar results were analyzed for bilayers with small thickness contrast ${ }^{22}$. However, these studies suggested unexpected complexity in describing high-order fold transitions $s^{3,9}$, and experimental access to these transitions has been restricted by limited strain that can be effectively applied with traditional loading methods. Actually, these studies reported only a part of wrinkle-fold transitions along a specific evolution route (either route 2 or 3 ). Our results feature three advancements over existing studies: i) a complete map of three buckling evolution routes, that is controllable transitions from wrinkles to $2^{n-1}$-folds and eventually to fractures, with $n=1,2$, or 3 depending on the soft microfilm thickness; ii) fractures of the stiff membranes with a preset pattern due to the quantized thickness dependence of the fracture period as well as the extreme stress concentration beyond the reach of traditional methods; ii) a concise scaling law to quantify the quantized wrinkles and factures.

Our strategy of controlling the buckling folds and fracture by peeling is powerful to tailor a series of largearea stiff membranes into microstructures with devisable shapes. Figure 4a shows an array of square $\mathrm{SiO}_{1.8}$ microflakes that are fabricated by sequentially peeling the stiff/soft bilayer along two perpendicular directions. The side length of the square microflakes can be fine-controlled to be $n \lambda_{0}(n=1$, 2 and 4 ) by varying $t_{\mathrm{s}}$ (Figs. $4 \mathrm{a}$ and $4 \mathrm{~d}$ ). Similar sequential fractures along two selected directions can yield rhombic microflakes (Fig. $4 \mathrm{~b}$ ). For the bilayer with $t_{\mathrm{s}}=t_{\mathrm{s} 1}$, both rectangular microflakes with a size of $1 \lambda_{0} \times 2 \lambda_{0}$ and square microflakes with a size of either $1 \lambda_{0} \times 1 \lambda_{0}$ or $2 \lambda_{0} \times 2 \lambda_{0}$ can be produced by sequential fractures along two perpendicular directions (Fig. 4c). Nevertheless, the specific size of formed microflakes is random at this critical point. Aside from the $\mathrm{SiO}_{1.8}$ membranes, our strategy is applicable to fracture many other brittle materials, as demonstrated by tailoring a polymethyl methacrylate (PMMA) membrane (Fig. S13a) into micro-ribbons. It can even be used to make an $\mathrm{Au}$ nanomembrane with topographical patterning at controlled scale (Fig. S13b), although brittle fracture cannot occur due to the high ductility of gold. The mechanically induced nano/micro-scale troughs in the Au film without using lithographic techniques may find potential applications in, for example, nanoparticle sieving ${ }^{6}$ and molecular manipulation ${ }^{27}$. Even monolayer graphene can be fractured into micro-/nano-ribbons (Fig. S13c), although more complex physical process may be involved here to induce the fracture, such as facture anisotropy ${ }^{28,29}$ and edge warping ${ }^{30,31}$.

We have developed a simple peeling strategy that can control the wrinkle-to-fold transitions with a preset period and then drive ordered fracture in stiff/soft bilayers. In principle, our strategy is applicable to 
fracture various two-dimensional materials of keen current interest. If technically matured, it will represent a new way for mass production of ribbons and flakes with customized edge orientations and nanometer sizes. For example, peeling a $\mathrm{MoS}_{2}$ monolayer $\left(t_{\mathrm{m}}=0.6 \mathrm{~nm}\right)$ resting on a PDMS microfilm with $t_{\mathrm{s}}=0.5$ $\mu \mathrm{m}$ from a rigid substrate would result in an array of nanoribbons with a uniform width of only $90 \mathrm{~nm}$ according to our scaling law. This mechanical tailoring method will be complementary to previous fabrication methods based on ion etching ${ }^{32,33}$ and chemical processing ${ }^{34-36}$. Moreover, the thicknessdependent wrinkle evolutions in stiff/soft bilayers may help us understand the ageing mechanism of skins as well as cerebral cortexes with strongly convoluted sulci and gyri that have proved crucial for achieving advanced intelligent functions ${ }^{14,15}$.

\section{References}

1. Bowden, N., Brittain, S., Evans, A. G., Hutchinson, J. W. \& Whitesides, G. M. Spontaneous formation of ordered structures in thin films of metals supported on an elastomeric polymer. Nature 393, 146-149 (1998).

2. Pocivavsek, L., Dellsy, R., Kern, A., Johnson, S., Lin, B. Lee, K. Y., \& Cerda, E. Stress and fold localization in thin elastic membranes. Science 320, 912-916 (2008).

3. Hutchinson, J. W. The role of nonlinear substrate elasticity in the wrinkling of thin films. Philos. Trans. A. Math. Phys. Eng. Sci. 371, 20120422 (2013).

4. Wang, C. G., Liu, Y. P. \& Tan, H. F. Global and local interactive buckling behavior of a stiff film/compliant substrate system. Int. J. Solids Struct. 102-103, 176-185, (2016).

5. Jiang, H. Q., Khang, D. Y., Song, J. Z., Sun, Y. G., Huang, Y. G. \& Rogers, J. A. Finite deformation mechanics in buckled thin films on compliant supports. P. Natl. Acad. Sci. USA. 104, 15607-15612 (2007).

6. Efimenko, K., Rackaitis, M., Manias, E., Vaziri, A., Mahadevan, L. \& Genzer, J. Finite deformation mechanics in buckled thin films on compliant supports. Nature Mater. 4, 293-297 (2005).

7. Huang, R. \& Suo, Z. Wrinkling of a compressed elastic film on a viscous layer. J. Appl. Phys. 91, 1135-1142 (2002).

8. Brau, F., Vandeparre, H., Sabbah, A., Poulard, C., Boudaoud, A. \& Damman, P. Multiple-length-scale elastic instability mimics parametric resonance of nonlinear oscillators. Nature Phys. 7, 56-60 (2010).

9 Nikravesh, S., Ryu, D. \& Shen, Y. L. Instabilities of Thin Films on a Compliant Substrate: Direct Numerical Simulations from Surface Wrinkling to Global Buckling. Sci. Rep. 10, 5728, (2020).

10. Kim, P., Abkarian, M. \& Stone, H. A. Hierarchical folding of elastic membranes under biaxial compressive stress. Nature Mater. 10, 952-957 (2011). 
11. Abi Ghanem, M., Liang X., Lydon B., Potocsnak L., Wehr T., Ghanem M., Hoang S., Cai S., \& Boechler N. Wrinkles Riding Waves in Soft Layered Materials. Adv. Mater. Interfaces 6, 1801609, (2018).

12. Zang, J., Zhao, X., Cao, Y. \& Hutchinson, J. W. Localized ridge wrinkling of stiff films on compliant substrates. J. Mech. Phys. Solids 60, 1265-1279 (2012).

13. Jin, L., Takei, A. \& Hutchinson, J. W. Mechanics of wrinkle/ridge transitions in thin film/substrate systems. J. Mech. Phys. Solids 81, 22-40 (2015).

14. Harmand, N., Huang, A. \& Hénon, S. 3D Shape of Epithelial Cells on Curved Substrates. Phys. Rev. $X 11,031028(2021)$.

15. Tallinen, T., Chung, J. Y., Biggins, J. S. \& Mahadevan, L. Gyrification from constrained cortical expansion. Proc. Natl. Acad. Sci. USA. 111, 12667-12672 (2014).

16. Budday, S., Steinmann, P. \& Kuhl, E. The role of mechanics during brain development. J. Mech. Phys. Solids 72, 75-92, (2014).

17. Almet, A. A., Byrne, H. M., Maini, P. K. \& Moulton, D. E. The role of mechanics in the growth and homeostasis of the intestinal crypt. Biomech. Model. Mechanobiol. 20, 585-608, (2021).

18. Khang, D. Y., Jiang, H. Q., Huang, Y. \& Rogers, J. A. A stretchable form of single-crystal silicon for high-performance electronics on rubber substrates. Science 311, 208-212 (2006).

19. Stafford, C. M., Harrison, C., Beers, K. L., Karim, A., Amis, E. J., VanLandingham, M. R., Kim, H. C., Volksen, W., Miller, R. D. \& Simonyi, E. E. A buckling-based metrology for measuring the elastic moduli of polymeric thin films. Nature Mater. 3, 545-550 (2004).

20. Mazaltarim, A. J., Bowen, J. J., Taylor, J. M. \& Morin, S. A. Dynamic manipulation of droplets using mechanically tunable microtextured chemical gradients. Nature Commun. 12, 3114 (2021).

21. Cao, Y. P. \& Hutchinson, J. W. Wrinkling Phenomena in Neo-Hookean Film/Substrate Bilayers. J. Appl. Mech. 79, 031019 (2012).

22. Auguste, A., Yang, J., Jin, L., Chen, D., Suo, Z. \& Hayward, R. C. Formation of high aspect ratio wrinkles and ridges on elastic bilayers with small thickness contrast. Soft Matter 14, 8545-8551 (2018).

23. Hillborg, H., Ankner, J. F., Gedde, U. W., Smith, G. D., Yasuda, H. K. \& Wikstrom, K. Crosslinked polydimethylsiloxane exposed to oxygen plasma studied by neutron reflectometry and other surface specific techniques. Polymer 41, 6851-6863 (2000).

24. Hohlfeld, E. \& Mahadevan, L. Unfolding the sulcus. Phys. Rev. Lett. 106, 105702 (2011).

25. Tari, H., Kinzel, G. L. \& Mendelsohn, D. A. Cartesian and piecewise parametric large deflection solutions of tip point loaded Euler-Bernoulli cantilever beams. Int. J. Mech. Sci. 100, 216-225 (2015). 
26. Zhao, R., Zhang, T., Diab, M., Gao, H. \& Kim, K. S. The primary bilayer ruga-phase diagram I: Localizations in ruga evolution. Extreme Mech. Lett. 4, 76-82, (2015).

27. Huh, D., Mills, K. L., Zhu, X., Burns, M. A., Thouless, M. D. \& Takayama, S. Tuneable elastomeric nanochannels for nanofluidic manipulation. Nature Mater. 6, 424-428 (2007).

28. Annett, J. \& Cross, G. L. Self-assembly of graphene ribbons by spontaneous self-tearing and peeling from a substrate. Nature 535, 271-275 (2016).

29. Zhang, T., Li, X., Kadkhodaei, S. \& Gao, H. Flaw insensitive fracture in nanocrystalline graphene. Nano Lett. 12, 4605-4610 (2012).

30. Alred, J. M., Zhang, Z., Hu, Z. \& Yakobson, B. I. Interface-induced warping in hybrid twodimensional materials. Nano Res. 8, 2015-2023 (2015).

31. Shenoy, V. B., Reddy, C. D., Ramasubramaniam, A. \& Zhang, Y. W. Edge-stress-induced warping of graphene sheets and nanoribbons. Phys. Rev. Lett. 101, 245501 (2008).

32. Lemme, M. C., Bell, D. C., Williams, J. R., Stern, L. A., Baugher, B. W. H., Jarillo-Herrero, P. \& Marcus, C. M. Etching of Graphene Devices with a Helium Ion Beam. ACS Nano 3, 2674-2676 (2009).

33. Ma, T., Ren, W. C., Zhang, X. Y., Liu, Z. B., Gao, Y., Yin, L. C., Ma, X. L., Ding, F. \& Cheng, H. M. Edgecontrolled growth and kinetics of single-crystal graphene domains by chemical vapor deposition. P. Natl. Acad. Sci. USA. 110, 20386-20391 (2013).

34. Jia, X. T., Hofmann, M., Meunier, V., Sumpter, B. G., Campos-Delgado, J., Romo-Herrera, J. M., Son, H. B., Hsieh, Y. P., Reina, A., Kong, J., Terrones, M. \& Dresselhaus, M. S. Controlled Formation of Sharp Zigzag and Armchair Edges in Graphitic Nanoribbons. Science 323, 1701-1705 (2009).

35. Sun, Z., Yan, Z., Yao, J., Beitler, E., Zhu, Y. \& Tour, J. M. Growth of graphene from solid carbon sources. Nature 471, 124-124 (2011).

36. Jiao, L. Y., Zhang, L., Wang, X. R., Diankov, G. \& Dai, H. J. Narrow graphene nanoribbons from carbon nanotubes. Nature 458, 877-880 (2009).

\section{Declarations}

\section{Data availability}

The data used in this study are available from the corresponding authors upon reasonable request. Source data are provided with this paper. 
Acknowledgments We thank Dr. Baowen Li, Luxian Li, Xuemei Li, Jianxin Zhou, Qin Wang and Jidong Li from NUAA for support in the experiments, Prof. Min Yi and Yufeng Guo from NUAA and Prof. Qingwen Li from Suzhou Institute of Nano-Tech and Nano-Bionics of CAS for help in the work. This work was supported by National Key Research and Development Program of China (2019YFA0705400), the National Natural Science Foundation of China (11772153), Natural Science Foundation of Jiangsu Province (BK20212008, BK20210271), the Research Fund of State Key Laboratory of Mechanics and Control of Mechanical Structures (MCMS-I-0421K01), the Fellowship of China national postdoctoral program for innovative talents (BX20200166), Postdoctoral Science Foundation of Jiangsu Province (2020Z355) and a Project Funded by the Priority Academic Program Development of Jiangsu Higher Education Institutions.

Author contributions W.G. leads the team, supervised the research and finalized the manuscript. Y.M. conducted the experiments. Y.M., J.Z. and W.G. performed the theoretical modeling. H.X. and W.G. performed finite-element simulations and analyses. H.X. drafted the discussion of simulation results. W.G., Y.M. and Z.Z. co-analyzed all data of the experiments and theories. L.L. assisted in the early experimental optimization. W.G., Z.Z. and Y.M. co-wrote the manuscript. All the authors provided helpful discussions.

Competing interests The authors declare that they have no competing interests.

\section{Additional information}

Supplementary information The online version contains supplementary material available at https://doi.org.

Correspondence and requests for materials should be addressed to W.G.

Reprints and permissions information is available online at www.nature.com/reprints.

\section{Figures}



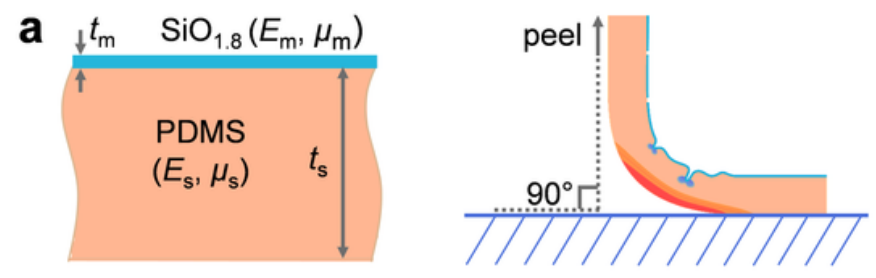

b
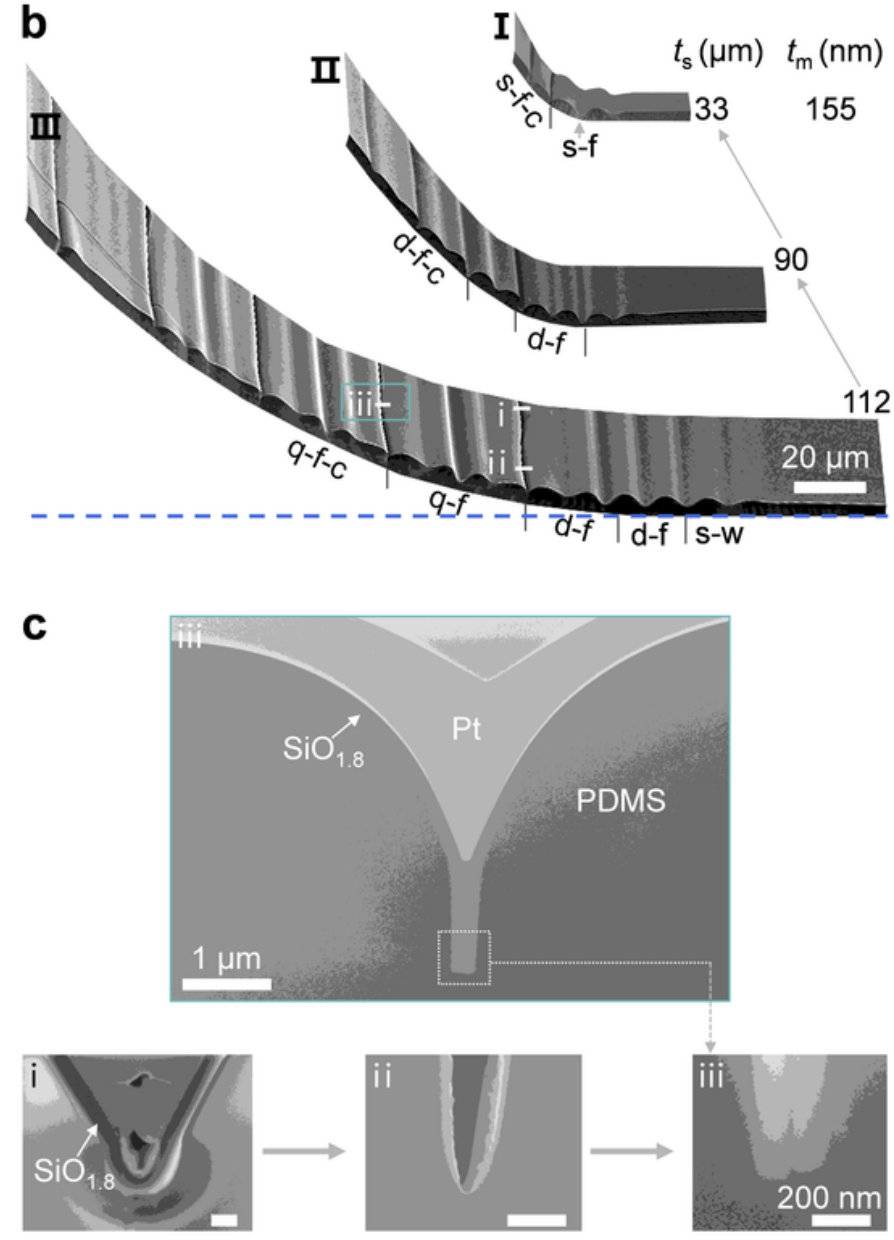
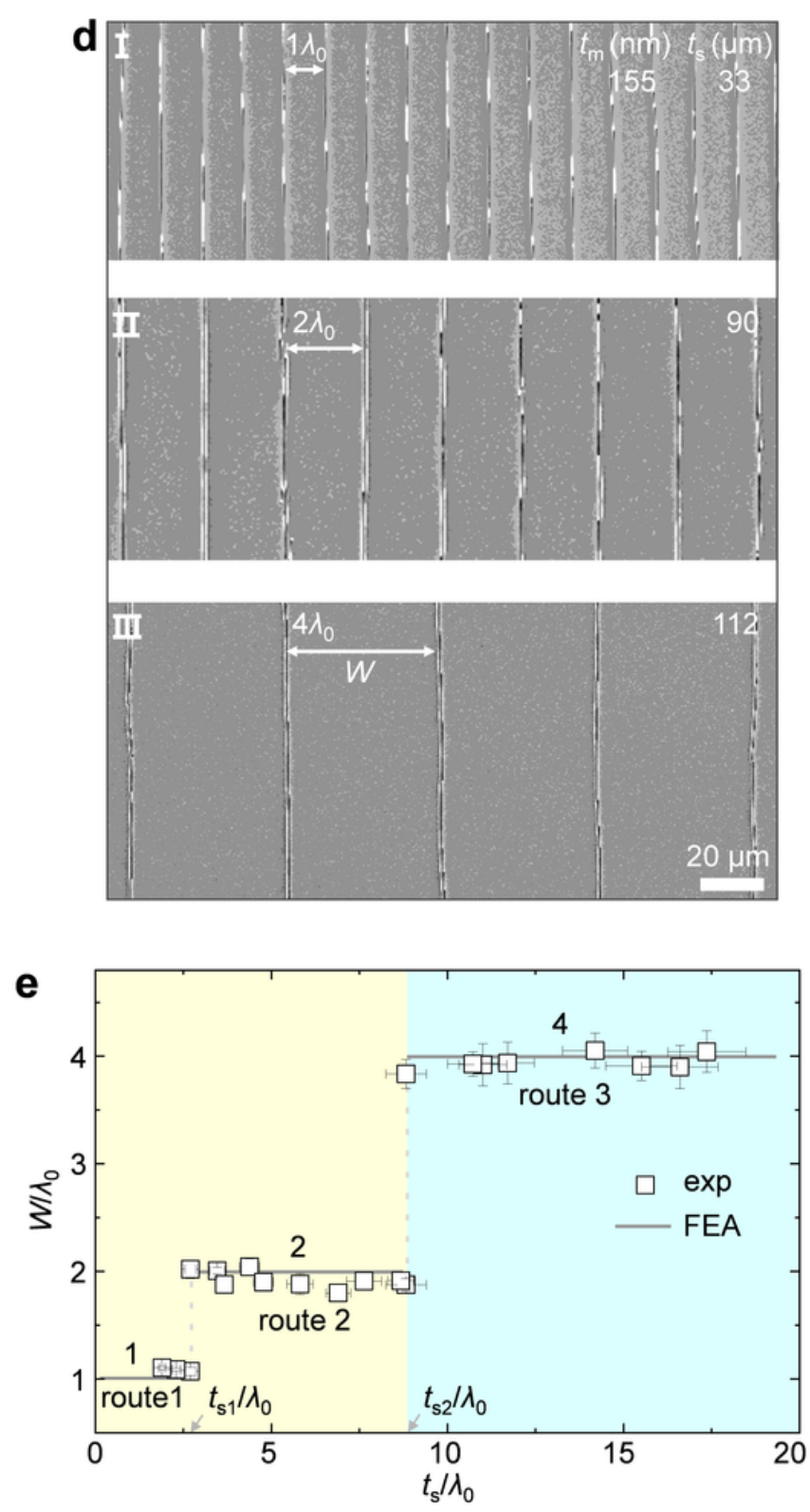

\section{Figure 1}

Controlled wrinkles and fracture in $\mathrm{SiO}_{1.8}$ /PDMS bilayers. a, Schematic illustration of the $\mathrm{SiO}_{1.8} / \mathrm{PDMS}$ bilayer and the peeling. $t_{\mathrm{m}}$ and $t_{\mathrm{s}}$ are the thicknesses of stiff $\mathrm{SiO}_{1.8}$ membrane and soft PDMS microfilm, respectively; the Young's modulus/Poisson ratio of the $\mathrm{SiO}_{1.8}$ membrane and the PDMS microfilm are 30 $\mathrm{GPa} / 0.25\left(E_{\mathrm{m}} / \mu_{\mathrm{m}}\right)$ and $1.8 \mathrm{MPa} / 0.48\left(E_{\mathrm{s}} / \mu_{\mathrm{s}}\right)$, respectively. $\mathbf{b}$, Side views of SEM images of buckling structures in three bending bilayers with different $t_{\mathrm{s}} \cdot t_{\mathrm{m}}=155 \mathrm{~nm}$ and $t_{\mathrm{s}}=33,90,112 \mu \mathrm{m}$ from top to bottom; s-w represents single wrinkle, while s-f, d-f, and q-f represent single, double, and quadruple folds, respectively; s-f-c, d-f-c and q-f-c represent fold-collapses in single, double, and quadruple folds, respectively; serial numbers $\mathbb{\otimes} \mathbb{\otimes}$ represent three mill locations of focused ion beams for characterizing cross-sections of collapsed folds in bending films. c, Images of focused ion beams taken at the locations $\mathbb{Q}-\mathbb{Q}$, illustrating the fracture process at collapsed folds. d, SEM images of fracture patterns in the three bilayers. $W$ denotes the width of fracture-produced micro-ribbons. e, Dependence of normalized fracture 
period $\left(W / \lambda_{0}\right)$ on $t_{s} / \lambda_{0}$. Squares represent experimental results and solid gray lines are numerical results; $t_{\mathrm{s} 1} / \lambda_{0}$ and $t_{\mathrm{s} 2} / \lambda_{0}$ are critical values of $t_{\mathrm{s}} / \lambda_{0}$ for the transition of fracture period; $\lambda_{0}=12.8 \mu \mathrm{m}$ for $t_{\mathrm{m}}=155$ $\mathrm{nm}$. Vertical bars represent standard error.
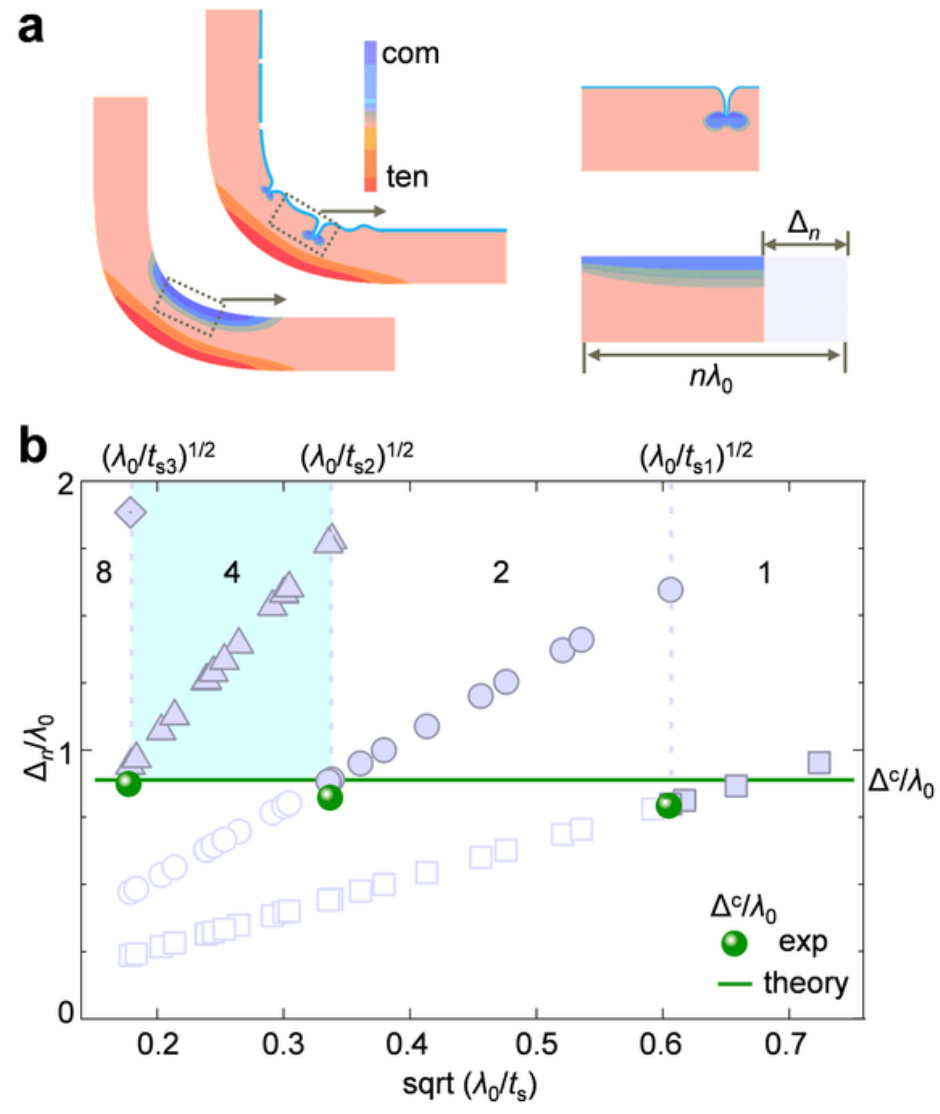
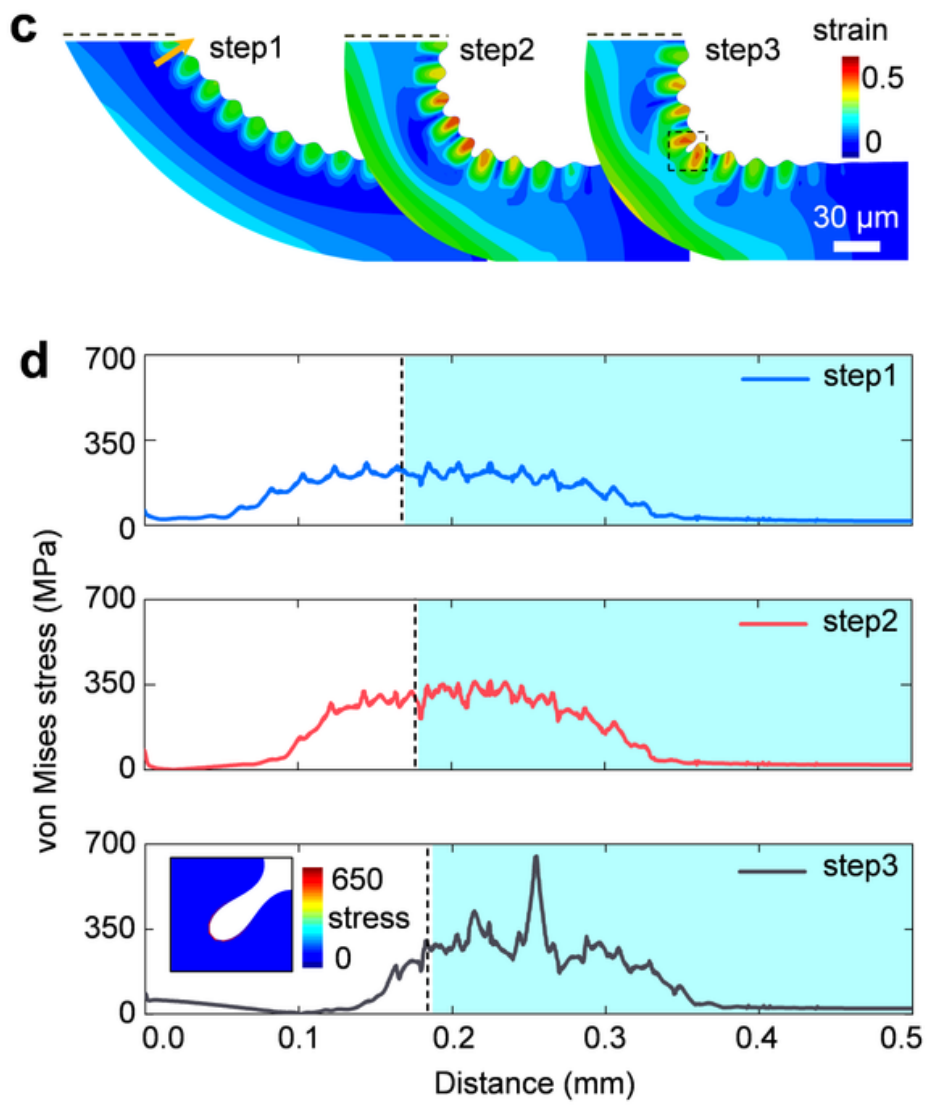

\section{Figure 2}

Mechanism of quantized wrinkles and fracture in $\mathrm{SiO}_{1.8} / \mathrm{PDMS}$ bilayers. a, Schematic illustration of a bent cantilever beam and compression-induced buckling in a bent bilayer. The shortened length of the microfilm $\left(\Delta_{n}\right)$ in one period length of the fold collapse is depicted on the right. The stress distribution in the beam is color-encoded. "com" and "ten" represent compressive and tensile stresses, respectively. $\mathbf{b}$, Normalized shortened lengths of the microfilm induced by single-fold collapse $\left(\Delta_{1} / \lambda_{0}\right.$, squares), doublefold collapse $\left(\Delta_{2} / \lambda_{0}\right.$, circles), and quadruple-fold collapse $\left(\Delta_{4} / \lambda_{0}\right.$, triangles) as functions of sqrt $\left(\lambda_{0} / t_{\mathrm{s}}\right)$; the critical shortened length for fracture occurrence $\Delta^{\mathrm{C}} / \lambda_{0}$ is about 0.9 by theory and 0.85 on average by experiments. c, Three-step illustrations of morphological evolution from wrinkles to double folds by finiteelement simulations. $\mathbf{d}$, Distributions of von Mises stress in the stiff membrane at the corresponding steps, with the stress concentration at the collapsed bottom highlighted in the inset. The dashed lines mark the positions indicated by dash lines in $\mathbf{c}$ and the shaded regions correspond to the visible parts of the bilayer in $\mathbf{c}$. The yellow arrow points to the displacement-controlled loading direction. 


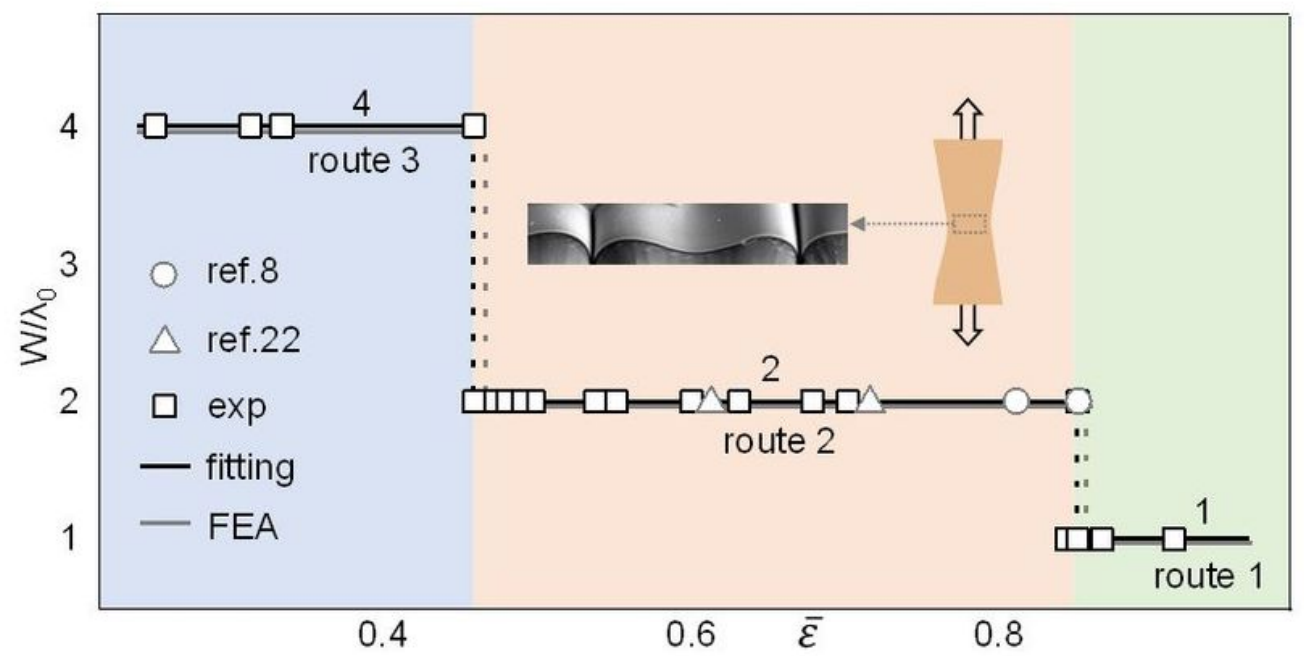

Fig. 3 | Generality of quantized wrinkles in bilayers by other loading methods. Dependence of normalized fracture ( or foldcollapse) period $\left(W / \lambda_{0}\right)$ on equivalent strain $(\bar{\varepsilon}), \bar{\varepsilon}=\varepsilon \lambda_{0} 10^{\frac{3}{2}} t_{\mathrm{m}}^{\frac{-1}{2}}$, where $\varepsilon$ is the lateral compressive strain. Gray circles and triangles show the data collected from references, in which different stiff/soft bilayers are directly compressed. Black line is fitting results of compressive experiments, while the gray line is converted from the data of peeling experiments shown in Fig. 1e.

\section{Figure 3}

See image above for figure legend.
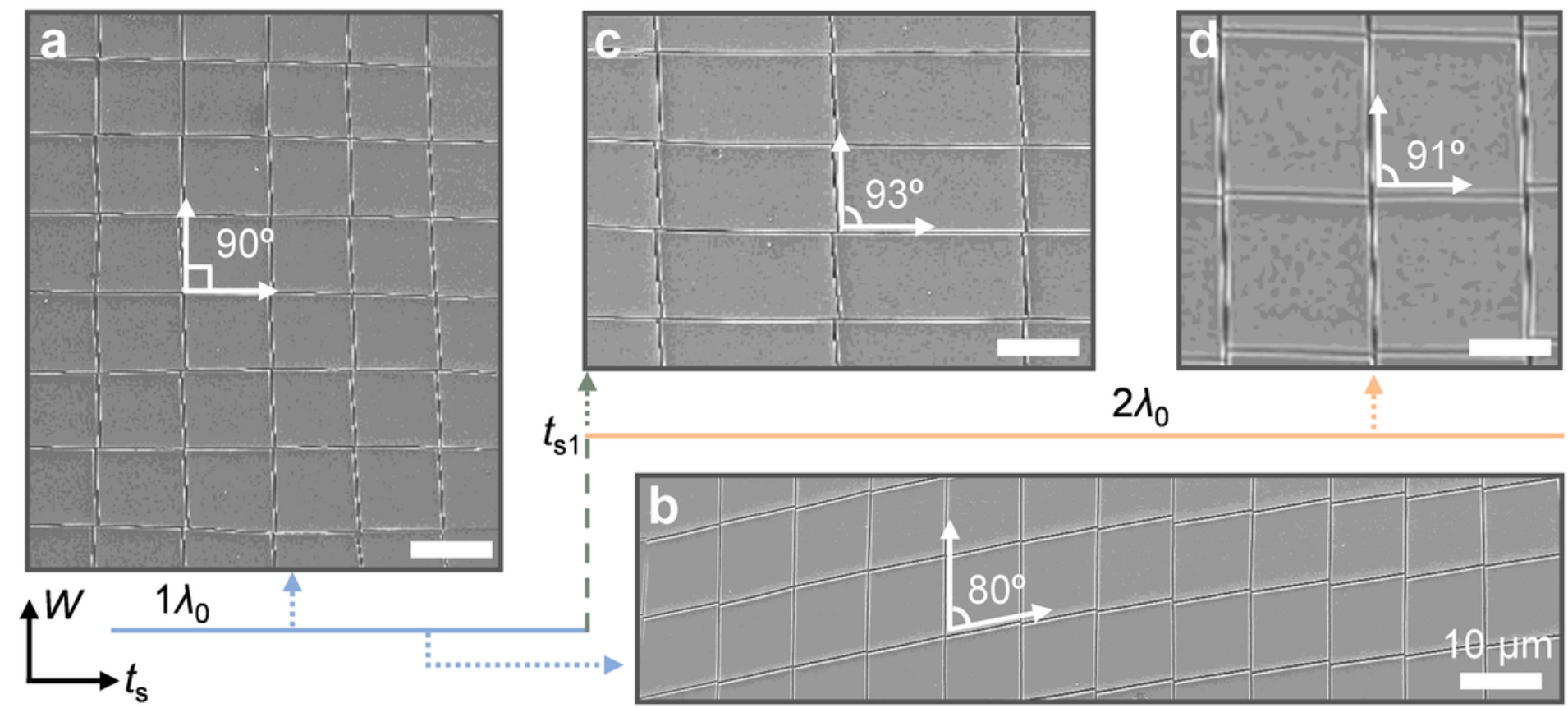

\section{Figure 4}

Application demonstrations of peeling-induced fracture of stiff/soft bilayers. a, An array of square $\mathrm{SiO}_{1.8}$ microflakes with a size of $1 \lambda_{0} \times 1 \lambda_{0}$. b, An array of rhombic $\mathrm{SiO}_{1.8}$ microflakes. $\mathbf{c}$, An array of rectangular 
$\mathrm{SiO}_{1.8}$ microflakes with a size of $2 \lambda_{0} \times 1 \lambda_{0}$, fractured from a bilayer with $t_{\mathrm{s}}=t_{\mathrm{s} 1} \cdot \mathbf{d}$, An array of square $\mathrm{SiO}_{1.8}$ microflakes with a size of $2 \lambda_{0} \times 2 \lambda_{0}$.

\section{Supplementary Files}

This is a list of supplementary files associated with this preprint. Click to download.

- Supplementaryinformation.docx 